LA-7885-MS, Vol. II

Informal Report

\title{
1-GWh Diurnal Load-Leveling Superconducting Magnetic Energy Storage System Reference Design Appendix A: Energy Storage Coil and Superconductor
}




\title{
1-GWh Diurnal Load-Leveling Superconducting Magnetic Energy Storage System Reference Design
}

Appendix A: Energy Storage Loil and Superconductor

\author{
R. I. Schermer
}
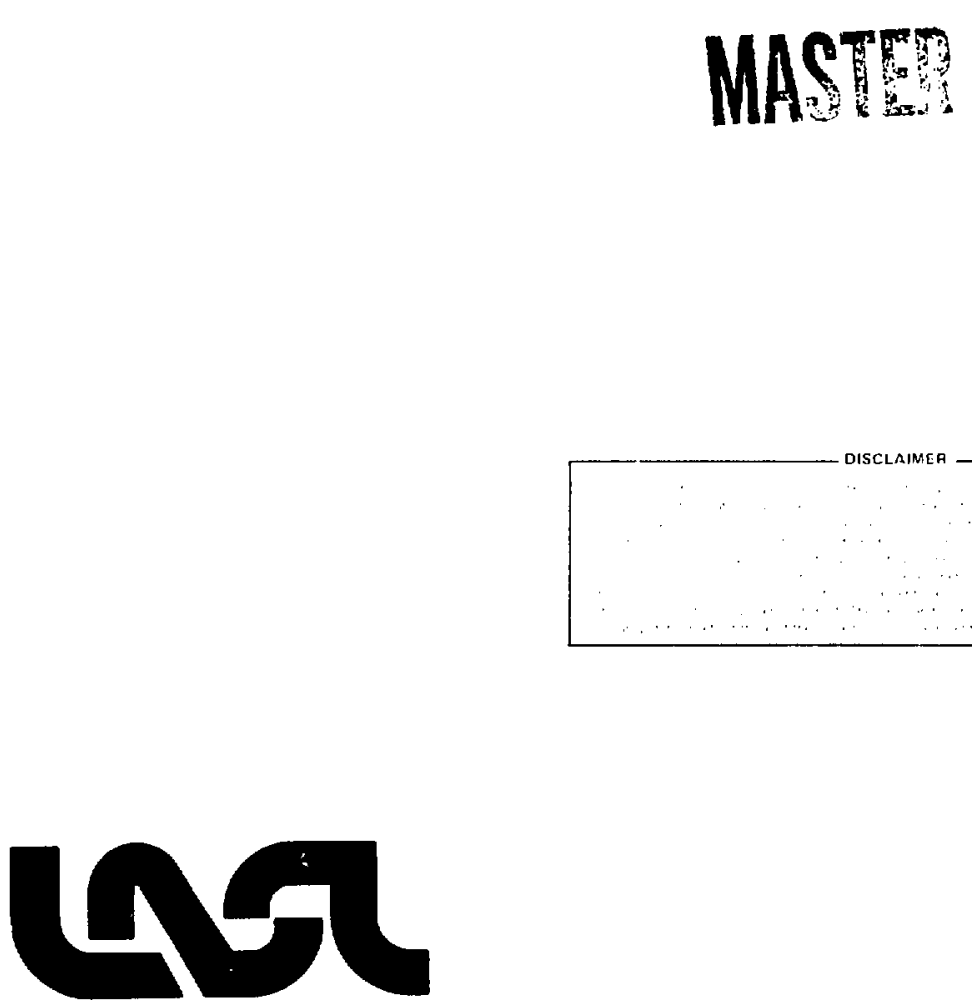


\title{
1-GWh DIURNA: LOAD-LEVELING SUPERCONDUCTING MAGNETIC ENERGY STORAGE SYSTEM REFERENCE DESIGN
}

APPENDIX A

ENERGY STORAGE COIL ANU SUPERCONDUCTOR

by

R. I. Schermer

\begin{abstract}
The technical aspects of a 1-GWh Superconducting Magnetic Energy Storage (SMES) coil for use as a diurnal load-leveling device in an electric utility system are presented. The superconductor for the coil is analyzed, and costs for the entire coil are developed.
\end{abstract}

I. INTRODUCTION

This appendix presents the details of the conceptual design for the coil and conductor of a 1-GWh Superconducting Magnetic Energy Storage (SMES) unit. The choices for total stored energy, coil shape, operating field, and operating current are discussed and justified. Once these free variables are chosen, the remainder of the coil parameters follows immediately and is given in Table A-I.

The proposed conductor is a 50-kA superconducting cable sandwiched between two parallel stabilizing elements, consisting of high-purity aluminum, jacketed with cold-worked copper for mechanical protection. Cable parameters are given in Table A-II. The allowable current density in the aluminum is set by coil protection considerations. Heat transport to the $1.8 \mathrm{~K}, 1-\mathrm{atm}$ coolant is 
discussed, and coolant channel dimensions are selected so that heat transfer at the conductor surface limits the allowable joule heating in the conductor.

TABLE A-I

PARAMETERS FOR 1-GWh COIL

Overall Parameters

$\begin{array}{ll}\text { Average coil radius } & 66 \mathrm{~m} \\ \text { Height } & 44 \mathrm{~m} \\ \text { Radial thickness } & 0.30 \mathrm{~m} \\ \text { Inductance } & 3170 \mathrm{H} \\ \text { Number of turns } & 4280 \\ \text { Winding pattern } & \text { pancake } \\ \text { Number of radial turns } & 5 \\ \text { Number of axial layers } & 856\end{array}$

Parameters of Axial Current Blocks

Block

Number

1

2 and 3

4 and 5

6 and 7

8 and 9

10 and 11

12 and 13

14 and 15

16 and 17

18 and 19

20 and 21

22 and 23
Axial

Turns

970

476

235

210

200

94

91

89

87

71

65

37

Height, m

8.8

$4 . n$

2.2

2.0

2.0

1.0

1.0

1.0

1.0

1.0

1.0

1.0
Mean Axial

Position, m

0

$\pm 6.6$

$+9.9$

$\pm 12.0$

$\pm 14.0$

$\pm 15.5$

$\pm 16.5$

$+17.5$

$\pm 18.5$

$\pm 19.5$

$\pm 20.5$

$\pm 21.5$
Axial

Turns/meter, $\mathrm{m}^{-1}$

22.0

21.6

21.4

21.0

20.0

18.8

18.2

17.8

17.1

14.3

13.0

7.4 
TABLE A-II

PROPERTIES OF 50-KA SUPERCONDUCTING COMPOSITE CABLE

$\begin{array}{ll}\text { Design } J_{C} \text { at } 1.85 \mathrm{~K}, 5.2 \mathrm{~T}, 10^{-14} \Omega-\mathrm{m} & 3.3 \times 10^{9} \mathrm{~A} / \mathrm{m}^{2} \\ \text { Alloy } & \mathrm{Nb}-46.5 \mathrm{wt} \% \mathrm{Ti} \\ \text { Operating current } & 50.0 \mathrm{kA} \text { at } 4.7 \mathrm{~T} \\ \text { Design critical current } & 55.6 \mathrm{kA} \text { at } 5.2 \mathrm{~T}\end{array}$

\section{Strand Parameters}

Design critical current

2417 A

Number of $f i l$ aments $\quad 1490$

Filament diameter

$25 \mu \mathrm{m}$

Cu-to-NbTi ratio

1.33

Diameter

$0.147 \mathrm{~cm}$

Twist pitch

$1.5 \mathrm{~cm}$

\section{Cable Parameters}

Number of strands

23

Packing factor

0.85

Dimensions

$0.277 \mathrm{~cm}$ by $1.67 \mathrm{~cm}$

Transposition length

$16.0 \mathrm{~cm}$

Mass per meter

$0.29 \mathrm{~kg} / \mathrm{m}$

After a consideration of magnetoresistance and cyclic-strain effects, the aluminum purity is specified. The final conductor design is given in Tables A-II and A-IV.

$A C$ losses in the conductor are considered next and shown to be nominal, with the possible exception of frictional lossas due to the relative motion of various coil components. This latter must be carefully controlled by proper coil construction. It is also shown that eddy current heating in the steel helium vessel will be extremely large unless the vessel contains an insulating section so that it does not act like a large conducting ring.

For reference design purposes the coil is constructed by placing individual radial and axial spacers between the conductor turns as the magnet is being wound. The mechanical design and cost of these spacers are considered in the final section. 
II. TOTAL STORED ENERGY AND DISCHARGE RATIO

The energy, $E$, to be exchanged with the electric utility has been specified as $1 \mathrm{GWh}$; but the energy, $W_{\max }$, actually stored in the magnetic field when the coil is fully charged has not been specified, nor has $W_{\text {min }}$. The three quantities are related by

$$
E=W_{\max }-W_{\min } .
$$

The discharge ratio, $\varepsilon$, is defined by the equation

$$
\varepsilon^{2}=W_{\max } / W_{\min },
$$

then

$$
\frac{E}{W_{\max }}=\frac{\varepsilon^{2}-1}{\varepsilon^{2}}
$$

The dc current and magnetic field at the limits of the charge-discharge cycle are related to $\varepsilon$ by

$$
\frac{B_{\max }}{B_{\min }}=\frac{I_{\max }}{I_{\min }}=\varepsilon
$$

If a SMES system is required to supply constant power to the utility grid, the ratio of coil terminal voltages at the limits of the cycle is also given by

$$
\frac{V_{\max }}{V_{\min }}=\varepsilon
$$

The installed converter power is independent of $V_{\max }$ for $2<\varepsilon<5$. A value of $\varepsilon=3.33$ is chosen as a reasonable compromise, because for any value of $\varepsilon$ greater than approximately three, the coil size decreases but little with $\varepsilon$, whereas the terminal voltage increases greatly. 


\section{OVERALL COIL DESIGN}

Choices for magnet shape have been considered in detail in previous work. ${ }^{1,2}$ A thin-walled solenoid is least expensive in terms of superconductor and easiest to support mechanicaliy but creates a rather large external field compared to a toroid. Options for using a guard coil are discussed in Chap. VIII ${ }^{3}$ and Ref. 1. A simple solenoid, as shown in Fig. A-1, is specified by three parameters: (a) the average radius, (b) the height, and (c) the radial thickness. For convenience, two ratios, $\alpha=c / 2 a$ and $\beta=b / 2 a$, are defined. In previous cost optimization studies, a broad cost minimum about $\beta=1 / 3$ is found, which is the value used here.

The operating magnetic field is also a result of a cost optimization procedure. ${ }^{1}$ A low field leads to more efficient superconductor use, lower capital cost for struts and refrigerator, and lower operating costs for refrigerator power. The cost of normal-conductor stabilizer changes very slowly with field; the details depend upon the magnetoresistivity of the stabilizer material. The sharpest field dependence, however, is that of the dewar, whose cost decreases dramatically with higher fields. The overall result is that the total cost tends to be very flat over a range of fields from 3 to $7 \mathrm{~T}$ even though there is a wide variation of unit costs for the various elements. If cold support is used, rather than warm support, the cost decreases slowly over the same field range. For the first iteration, a value of $4.5 \mathrm{~T}$ was chosen. The coil radius, $a$, was then fixed by the requirement that a single-layer solenoid with uniform current density and $\beta=1 / 3$ would store energy $W_{\max }$ with a maximum field, $B_{\max }$, at the winding of $4.5 \mathrm{~T}$.

$$
W_{\max }=\frac{B_{\max }^{2}}{2 \mu_{0}} f(B) a^{3},
$$

where the factor $f(\beta)$ was obtained by numerical computation. The design then evolved through several iterations and stages of complexity in which the fixed parameters were taken as $W_{\max }$, $B$, the value of "a" just calculated, and a winding thickness of $30 \mathrm{~cm}$. During the process, the values of $B_{\max }$ remained in the range of 4 to $5 \mathrm{~T}$; therefore, the adjustment of the value of "a"was unnecessary.

For a thin-walled solenoid $\alpha \rightarrow 0$, the magnetic field tends to peak sharply at the coil end unless the current density is allowed to vary with 
axial position. A calculation was performed in which axial spacing of turns was varied in 24 discrete steps. This results in a magnetic field which is constant to within a few percent everywhere on the innermost conductor turns. The winding parameters from this calculation are given in Table A-I. There is no correlation between the height of the various current blocks in Table A-I and the height of the segments into which the coil is mechanically divided for stress reasons. The design is purely illustrative. A further iteration will be necessary because " $c$ " will change significantiy from its assumed value, and the current blocks will have spaces between them.

\section{MAXIMUM CURRENT AND TERMINAL VOLTAGE}

The power rating of a SMES unit is determined by the application, but the separate choice of current and voltage is one of engineering judgment. The installed converter cost is almost independent of voltage over a wide range covering typical power system practice. Possibly there is an optimum current which results in a minimum cost for the total system. At this point, reasonable power system voltage and current have been chosen for the reference design because industry must acquire considerable experience in the field construction of large coils before a valid optimization can be performed. For reference, several factors which can enter into ar optimization are listed below.

(a) The higher the current, the more difficult the conductor will be to handle physically. Thus, the conductor winding rate will be slower while the length of conductor to be laid down decreases inversely as the current. The cheapest coil may use the largest possible conductor.

(b) The higher the current, the smaller the terminal voltage will be. one-half the terminal voltage appears between each magnet terminal and the grounded parts of the dewar. The maximum safe value for this potential difference to ground can only be conjectured. Superconducting power transmission line designs call for $>100 \mathrm{kV}$. For a 1-GWh 250-MW SMES unit to succeed, development must proceed to the point where voltages of at least 50 to $100 \mathrm{kV}$ are allowable under either routine operation or fault: conditions.

(c) A drawback to high current is the extra refrigeration required by the power leads. Although this is a minor contribution for the main pair 
of leads, it may be significant if multiple leads are required for protection.

(d) At some sufficiently high current, a single layer coil that has certain mechanical advantages can be built. For this design, multiiayer coils are considered together with the structural problems involved in their construction, rather than to limit the design to singlelayer coils at this stage. A modular conductor probably can be constructed for a high enough current to allow single-layer construction if necessary. At this stage, conductors which cannot support stresses of the order of $10^{8} \mathrm{MPa}(15 \mathrm{ksi})$ in any direction seem extremely inconvenient to use in any coil design.

(e) A small amount of stabilizer can be saved by running at a considerably lower current and correspondingly higher terminal voltage.

\section{v. STABILITY AND PROTECTION}

Stability refers to the capacity of a superconducting system to undergo a perturbation and maintain or regain the superconducting state. The proposed conductor is designed so that a long length of conductor may be driven well above its transition temperature and recover, that is, return to the superconducting state. The actual stability limit of the system is also determined by details of helium inventory and fluid flow which affect local helium replenishment in addition to the conductor properties.

A cryostable conductor can, in principle, carry a current density of more than $50 \mathrm{kA} / \mathrm{cm}^{2}$, which consequently reduces the cost of stabilizer to a trivial amount. Such a design would take advantage of the extremely good heat transfer available in liquid helium at $1.8 \mathrm{~K}$ and 1.0 -atm pressure and the low electrical resistance in a magnetic field of high-purity aluminum.

In practice, however, the question of protection intrudes upon this idealized scheme. Protection is the process whereby the energy is removed from a coil to prevent damage from occurring. Damage may result from overheating and its attendant thermal stresses or from electrical breakdown. Three distinct types of events are generally cited as requiring protective action.

(a) Design or construction flaws could lead to conditions requiring emergency action. This is the most common historical reason for coil failure. A proper engineering development program should reduce the probability of such occurrences. 
(b) The conductor could lose its cryostability so that if it went normal it would not recover automaticaily. Blockage of coolant channels is of ten cited as a possible hazard in this regard.

(c) Unforeseen conditions such as reirigerator failure or vacuum leaks might force emergency action.

Protection involves providing sufficient time to discharge the coil energy; the time is lengthened by lowering the current density in the conductor stabilizer and hence raising the stabilizer volume. This process becomes prohibitively expensive if it is thought necessary to discharge the entire coil at a modest turn-to-turn voltage. Clearly it is necessary to perform a cost-benefit analysis on a protection system. To perform such a calculation the probabilities and consequences of various failure modes must be known.

The present level of design leaves some uncertainties to be resolved. A design that attempts to account for all current unknowns may be unrealistical$7 y$ conservative by a 1 arge factor. To ignore the protection problem is certainly optimistic. A micdle course, in which an emergency requires protecting only one segment o: the magnet, is postulated. If $1 \%$ of $W_{\max }$ is to be removed with a turn-to-turn voltage of $100 \mathrm{~V} / \mathrm{turn}$, the maximum allowable current densities are $21 \mathrm{kA} / \mathrm{cm}^{2}$ in copper or $15 \mathrm{kA} / \mathrm{cm}^{2}$ in aluminum. The stability analysis must then be used to determine if such current densities can indeed be tolerated. The answer depends greatly upon the details of the cryogenic and structural design. Under the assumptions that will be made, copper cannot be operated readily at this high a current density; and there is no great cost advantage for running aluminum at so high a density. Reducing the current density is worthwhile, and it eases the protection problem.

\section{CONDUCTOR DESIGN}

\section{A. General Considerations}

The conductor should be fully cryostable, exhibit modest ac losses, should be able to be fabricated in relatively long lengths and to be wound in place underground, and should support a reasonable stress level. The conductor chosen is stabilized by aluminum with its current density set at $15 \mathrm{kA} / \mathrm{cm}^{2}$ for protection reasons. A sample of copper-jacketed, aluminum-stabilized conductor of the type specified here has been prepared by Airco Superconductors, Inc. for the SMES program. Results of a cost study on high-purity aluminum, performed by Alcoa, are given in Appendix B. ${ }^{4}$ 
For convenience in fabrication and handling, the conductor consists of two parallel elements in electrical contact sandwiched about a 13-strand superconducting composite cable. Such a moduiar design would allow grading the quantity of superconductor and the shape and quantity of stabilizer as a furction of location. This would result in significant material savings in these elements as well as in the interturn spacers.

\section{Superconductor Design}

Cable parameters are given in rable A-II. The conductor is sized to operate at $90 \%$ of the short-sample critical current along the maximum field load ine in the coil. If the critical current is measured at $1 \times 10^{-14} \mathrm{sm}$, there will be at least an additional 10\% safety margin in the magnet operation in that the Joule heating will be unnoticeable at this resistance level. Thus, each cable strand must operate at $2.17 \mathrm{kA}$ at $4.7 \mathrm{~T}$ but is sized to carry $2.42 \mathrm{KA}$ at $5.2 \mathrm{~T}$.

The appropriate vaiue of expected critical current density at $1.85 \mathrm{~K}$ must be caiculated. There are few data for guidance nor is there a sufficientiy aciurate theory. In general

$$
J_{c}(T) \infty 1-\left(\frac{T}{T_{c}}\right)^{n} \quad=
$$

where $n$ is of the order of 1 to 2. The data of Hancox shows that $J_{c}(1.85) / J_{C}(4.2)=1.6$ at $3 \mathrm{~T}$, for which $T_{c}=7.8 \mathrm{~K}$. From Eq. $(A-1)$, the ratio is expected to lie between $1.32(n=2)$ and $1.65(n=1)$. The data thus lie rather close to a linear relation, as do data above $4.2 \mathrm{k}$. With $n=1$ and $T_{2} ? 1.0 \mathrm{~K}$, corresponding to $5 \mathrm{~T}$,

$$
\frac{J_{C}(1.85 \mathrm{~K})}{J_{C}(4.2 \mathrm{~K})}=1.84 \text { at } 5 \mathrm{~T} \text {. }
$$

The ratio might be even higher for an alloy whose fabrication is optimized for the specified conditions. For a typical value of $J_{c}(4.2 \mathrm{~K}, 5.2 \mathrm{~T})$ $=1.8 \times 10^{9} \mathrm{~A} / \mathrm{m}^{2}, \quad J_{c}(1.85 \mathrm{~K}, 5.2 \mathrm{~T})=3.3 \times 10^{9} \mathrm{~A} / \mathrm{m}^{2}$. With a Cu-to$\mathrm{NbTi}$ ratio of 1.33, the composite area is small enough that a monolith could be used, but a monolith does not allow the amount of superconductor to be varied simply as a function of position. The superconducting composite should be 
mounted on a vertical face of the stabilizer where it is subject only to the modesi radial compressive stress, rather than on a horizontal $f$ ace where it must support the large axial stress. It also yields smaller ac losses when mounted vertically. For these reasons a 23-strand cable, to be set into shallow channels in the stabilizer faces, is specified with an aspect ratio of six.

The strand called for in Table A-II is typical of those presentily being produced commercially. A single extrusion billet yields at least $180 \mathrm{~kg}$ or $14000 \mathrm{~m}$ of strand in several long lengths. Cable is typically fabricated from random lengths cold welded together, with the cold welds staggered along the cable length, so that there is little waste and no maximum length restriction.

The coil requires $1.77 \times 10^{6} \mathrm{~m}$ of $55.6-\mathrm{kA}$ conductor or $9.9 \times 10^{7} \mathrm{kA}-\mathrm{m}$ of conductor. From Table 4.2 of kef. 5, the composite can be estimated to cost $\$ 1.10 / \mathrm{kA}-\mathrm{m}$ at $4.2 \mathrm{~K}$ and $5 \mathrm{~T}$ and hence should cost $\$ 0.60 / \mathrm{kA}-\mathrm{m}$ at $1.85 \mathrm{~K}$ and $5 \mathrm{~T}$. The total price of the composite strands is then $\$ 59.3 \mathrm{million}$, or a saving of $\$ 41.6$ million over the cost if the coil were to operate at $4.2 \mathrm{~K}$. There is an additional cost of $\$ 0.60 / \mathrm{m}$ of $23-\mathrm{strand}$ cable for cabling, or $\$ 1.06$ million.

The above design puts far too much superconductor in the low-field regions of the coil. Approximately 29\% of the superconductor could be eliminated if the Cu-to-NbTi ratio was adjusted so that just enough NbTi alloy is located at every position to operate at $90 \%$ of critical current. The most straightforward way to do this is to replace composite cable strands by pure copper strands. This replacement also disrupts the perfect cable transposition and results in nonuniform current distribution among the strands. A second alternative is to fabricate cable strands with ten different Cu-to-NbTi ratios, which could be phased into the cable as desired. The overall composite conductor cost is thus estimated at $71 \%$ of the previously quoted value, or $\$ 42.1$ million for the strands plus $\$ 1.06$ million for cabling.

There is considerable margin for decrease in the above composite price, which represents a cost of $\$ 350 / \mathrm{kg}$ of contained $\mathrm{NbTi}$ compared to the current price of $\$ 100 / \mathrm{kg}$ for the alloy as fabricated into rods ready to be stacked and extruded. The coil will require roughtly 2800 extrusion billets, compared to the 1 argest order the industry has previously processed of 100 billets for the Fermi National Accelerator Laboratory (FNAL). This represents a considerable margin for development to reduce the cost of the finished conductor. 


\section{CRYOGENIC DESIGN CONSIDERATIONS}

\section{A. Introduction}

The conductor must recover from perturbations which drive it normal by transferring heat to the liquid helium. There are two mechanisms which limit the steady-state heat removal capacity. First, there is a maximum heat flux that can leave a surface. The assumption is usually made that the maximum value is set by the peak nucleate boiling flux (PNBF), although this assumption is in fact quite conservative for localized perturbations. The PNBF in He II at $1 \mathrm{~atm}$ is at least $5 \mathrm{~W} / \mathrm{cm}^{2}$. A limit of $5 \mathrm{~W} / \mathrm{cm}^{2}$ is assumed for this design. A second condition is set by the limiting heat flux, $q_{l}$, in the coil cooling channels. Experiments have verified that $q_{\ell}$ is a function of the channel length, $\ell$. If $l$ is measured in centimeters, then

$$
q_{2}=\frac{q_{1}}{2^{1 / 3}} \quad W / \mathrm{cm}^{2},
$$

where $q_{\ell}$, the limiting flux in a channel $1 \mathrm{~cm}$ long, is a function of the temperature at the cold end of the channel and of the bath pressure, and is approximately $7.5 \mathrm{~W} / \mathrm{cm}^{5 / 3}$ for a bath at $1.85 \mathrm{~K}$ and $1 \mathrm{~atm}$. Because the total heat which can be carried by a channel is found by multiplying $q_{\ell}$ by the cross-sectional area of the channel, narrow, internal channels between subconductors make a negligible contribution to heat transport when compared to the far wider channels between coil turns. Thus, internal channels have been completely el iminated in the conductor design.

If more than one conductor adjacent to a particular channel should become normal, the allowable heat generation rate from each conductor would have to be reduced because the channel could carry away less heat from each conductor. This condition affects the economics because it requires use of a higher purity aluminum. Such an event is too unlikely to be used as a design basis. A conductor is presumably driven normal by a local perturbation that causes a few meters of conductor to be in the normal state for a few hundred milliseconds. In a properly designed coil this would be a rare occurrence and the chance of its happening in two adjacent conductors should be even more rare. Further, the limitation of the maximum design heat flux to the value, which can be carried without a transition to film boiling, is also 
conservative. Thus, the cryogenic design will only consider an event in which one isolated length of conductor must recover.

Equation (A-2) represents the experimental data for a single, fluid-filled channel, but it is not known how it applies to a coil in which there are multiple, interconnected channels between the presumed normal conductor and the bulk of the He II liquid. The assumption is made that, once the heat has left the immediate vicinity of the conductor, there are so many parallel heat conduction paths that the winding effectively acts like an open bath. Eventual$1 y$, however, experiments must be performed on a mockup of the structure.

The detailed geometric arrangement of the conductors affects cost both through its effect upon heat transfer and upon the structural design. The optimum design will most likely be different from that presented here but cannot now be determined because of a lack of experimental and cost data.

B. Channel Design

The channels will be made large enough so that the PNBF at the conductor surface will be the controlling parameter. Consider the conductor array shown in Fig. A-2, in which the central conductor is assumed to be normal. From symmetry considerations only one-quarter of the conductor, which carries $12.5 \mathrm{kA}$ in a block of stabilizer of vertical height $w$ and radial thickness $t$, need be considered. The resulting Joule heating is

$$
q_{J}=\frac{I^{2} \rho}{A}=\frac{I^{2} \rho}{W t} \quad W / c m
$$

This heat is removed through a horizontal channel of height $x^{\prime \prime}$ and length $t$ and a vertical channel of width $X^{\prime}$ and height $W^{\prime}$. Each channel is only open for a fraction, $f$, of its length into the $p$ lane of the figure with the remainder of the channel blocked by structural material. From Eq. (A-2), it follows that the maximum power which can be removed per centimeter of conductor by the horizontal channel is

$$
q_{H}=\frac{7.5 X " f}{t T / 3} w / c m,
$$


whereas for the vertical channel it is

$$
q_{v}=\frac{7.5 \times x^{\prime} f}{w^{1 / 3}} w / c m
$$

With the peak surface heat flux set at $5 \mathrm{~W} / \mathrm{cm}^{2}$, the maximum power which can enter the horizontal channel per centimeter of conductor is

$$
q_{H}^{\prime}=5 \mathrm{tf} \mathrm{W/cm} \text {, }
$$

and for the vertical channels

$$
q_{v}^{\prime}=5 w f w / c m .
$$

The requirements $q_{H}>q_{H}^{\prime}$ and $q_{V}>q^{\prime}{ }_{V}$ lead to the conditions

$$
x^{\prime \prime} \geq 0.67 t^{4 / 3} \text { and } X^{\prime} \geq 0.67 w^{4 / 3} \text {. }
$$

Furthermore, with

$$
\begin{aligned}
& q_{V}^{\prime}+q_{H}^{\prime}=q_{J}, \\
& 5 f(w+t) \geq q_{J} .
\end{aligned}
$$

Axially, the coil turns are not required to be very close together even at the center of the coil, so there are horizontal spaces which must be filled in any event. See Table A-I. Suppose $k>t$ to assist in filling these spaces. Then Eq. $(A-4)$ demands that the horizontal separation, $X^{\prime}$, be 1 arge, so that otherwise extraneous material must be included in the vertical spacers. Also, the axial force must now be supported by the smaller bearing surface, $t$, which may require a larger fraction, $f$, of the horizontal channels to be obstructed.

Another option would be to make $t \gg w$. This requires narrow vertical channels with a saving on extraneous material and large horizontal channels, which exist anyway. To the extent that the horizontal channels would be larger than necessary, this again represents extraneous spacer material. Now, 
however, the bearing load is more widely distributed, which might allow a larger fraction of the channel to be left open.

To obtain numerical results for minimum channel sizes, the value of $q_{j}$ will be calculated in the following section.

C. Stabilizer Design

If the conductor aspect ratio, $r$, is defined by

$$
\xi=\frac{w}{t},
$$

then Eqs. $(A-3),(A-5)$, and $(A-6)$ yield a relation between the conductor dimensions and the required resistivity which is

$$
\mathrm{t}^{3} \geq \frac{\mathrm{I}^{2} \rho}{5(1+\xi) f}
$$

For the present design, a square conductor with $\xi=1$ is used, although it is likely economic optimization may require a different value.

Equation $(A-7)$ also implies a maximum value of current density for a given $\mathrm{n}$ and $\xi$. For $\xi=1$ the relation becomes

$$
J=\frac{I}{t^{2}}=\left(\frac{10 f}{\rho}\right)^{2 / 3} I^{-1 / 3}
$$

Table A-III gives the conductor dimension and resistivity for various values of $J$ and $f$ as calculated with Eqs. $(A-7)$ and $(A-8)$.

For annealed oxygen-free copper at $4.7 \mathrm{~T}, \rho=2.2 \times 10^{-8} \Omega \mathrm{cm}$. Since this material has a yield strength of only $10 \mathrm{ksi}$ at $4.2 \mathrm{~K}$, a more useful stabilizer might be given 5 to $7 \%$ cold work. Cold work raises the yield strength to $>30 \mathrm{ksi}$ but only raises the resistivity to $2.97 \times 10^{-8} \Omega \mathrm{cm}$. Table A-III reveals that in either case, with $f \approx 0.5$, as will probably be required in the most highly stressed coil regions, it will be necessary to operate a copper-stabilized coil at $<15 \mathrm{kA} / \mathrm{cm}^{2}$. That is, for copper it is more difficult to meet the heat transfer requirement of Eq. $(A-5)$ than the protection requirement. For aluminum the resistivities given in Table A-III are perfectly reasonable and there seems to be a sizable cost advantage in using aluminum, at least in terms of the raw materials involved. 
D. Properties and Costs of Stabilizing Materials

Table A-III gives the overall current density and the resulting required value of $\rho$ for the conductor. It is still necessary to calculate $J$ and $\rho$ separately for the aluminum and the copper jacket.

The relations

$$
\left(\mathrm{J}_{\rho}\right)_{\mathrm{Al}}=\left(\mathrm{J}_{\rho}\right)_{\mathrm{Cu}}=\mathrm{J} \rho
$$

and

$$
J A=\Sigma_{i}(J A)_{i}
$$

imply that

$$
\frac{A}{\rho}=\sum_{i}\left(\frac{A}{\rho}\right)_{i}
$$

The assumption is made that $A_{C u} / A=0.2$ and $A_{A} / A=0.8$, which corresponds to the smallest copper fraction that $A$ irco has yet attempted to rabricate, and $\rho_{\mathrm{Cu}}=2.97 \times 10^{-8} \Omega \mathrm{cm}$. There remains one free variable among $\rho_{A l}, \mathrm{~J}_{\mathrm{Al}}$, and $\mathrm{J}_{\mathrm{Cu}}$.

TABLE A-III

DESIGN PARAMETERS FOR A 50-KA CONDUCTOR MODULE

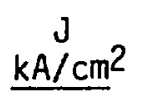

20

15

10

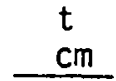

0.791

0.913

1.12

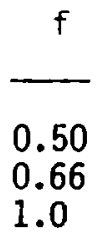

0.50

0.66

1.0

0.50

0.66

1.0 $\underline{10^{-8}}{ }^{\rho} \Omega-\mathrm{cm}$

1.58

2.11

3.16

2.44

3.24

4.87

4.47

5.96

8.94 
The cross-sectional area of stabilizer required is proportional to $1 / \mathrm{J}$ and, therefore, to $\rho^{2 / 3}$. The average price of high-purity aluminum is expected to vary approximately as $p^{-0.6}$. The total cost of aluminum stabilizer is, therefore, expected to decrease only very slowly with resistivity; thus the

Cost of Al $\sim \rho^{0.07}$.

This implies that there is no gat economic advantage in running an al1aluminum coil at the maximum $\mathrm{J}$ allowed by protection considerations. By contrast, the cost per kilogram of copper siabilizer is essentially independent of its resistivity so that a copper stabilizer will be cheapest when run at its maximum J. For the copper-jacketed aluminum stabilizer, the situation is rather more complex. The copper area is giver, by

$$
A_{C u}=0.2 A=0.2 \frac{I}{J}
$$

The copper area and hence the copper cost will thus be minimized by maximizing $J$ consistent with all the other constraints in the problem. For the particular case chosen, the limiting condition is $J_{A l}=15 \mathrm{kA} / \mathrm{cm}^{2}$. The operational parameters of the conductor are now fully determined and are given in Table A-IV. The conductor is shown in Fig. A-3.

The cost of aluminum depends upon its residual resistivity ratio (RRR) in the unstrained, zero-field condition. Segal ${ }^{6}$ has shown that a cyclictensile strain of $0.1 \%$ produces a change of $20 \%$ in the RRR of high-purity aluminum within the first thousand cycles and little change thereafter. In the current application, the material is being compressed axially while being restrained radially and longitudinally, and it is likely that a stress-strain curve will be virtually linear for strains up to and beyond $0.1 \%$. Only small energy losses are expected from mechanical hysteresis with perhaps only minor changes in RRR. Clearly, experimental work is necessary to resolve these matters. Meanwhile, a maximum tolerable strain of $0.1 \%$ in the aluminum and a corresponding increase of $20 \%$ in the electrical resistivity are assumed. The results of Fickett $^{7}$ indicate that the resistivity in a field of $4.7 \mathrm{~T}$ is approximately 2.6 times that in zero field for an initial RRR of 1000 . The assumption is made that the aluminum in the conductor will have its 


\begin{tabular}{|c|c|}
\hline \multicolumn{2}{|c|}{ OPERATIONAL PARAMETERS OF A 50-kA STABILIZER } \\
\hline Dimensions & $1.856 \mathrm{~cm}$ by $1.856 \mathrm{~cm}$ \\
\hline Aluminum dimensions each side & $0.80 \mathrm{~cm}$ by $1.73 \mathrm{~cm}$ \\
\hline Copper jacket thickness & $0.065 \mathrm{~cm}$ \\
\hline Volume fraction aluminum & 0.8 \\
\hline 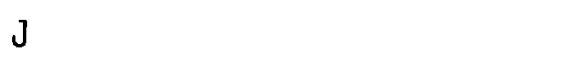 & $14.5 \mathrm{kA} / \mathrm{cm}^{2}$ \\
\hline$J_{A 1}$ & $15.0 \mathrm{kA} / \mathrm{cm}^{2}$ \\
\hline $\mathrm{J}_{\mathrm{Cu}}$ & $12.5 \mathrm{kA} / \mathrm{cm}^{2}$ \\
\hline$\rho$ & $2.56 \times 10^{-8} \Omega \mathrm{cm}$ \\
\hline${ }^{P_{A 1}}$ & $2.47 \times 10^{-8} \Omega \mathrm{cm}^{\mathrm{a}}$ \\
\hline$\rho_{A 1}$ & $7.97 \times 10^{-9} \Omega \mathrm{cm}^{\mathrm{b}}$ \\
\hline$\rho_{\mathrm{Cu}}$ & $2.97 \times 10^{-8} \Omega \mathrm{cm}$ \\
\hline Mass of aluminum in magnet & $1.27 \times 10^{6} \mathrm{~kg}$ \\
\hline Mass of copper in magnet & $1.05 \times 10^{6} \mathrm{~kg}$ \\
\hline
\end{tabular}

resistivity increased by an overall factor of $1.2 \times 2.6=3.1$ from its original value. The original aluminum, therefore, is required to have a resistivity of less than $7.97 \times 10^{-9} \Omega \mathrm{cm}$ or an RRR of 340 . From the Alcoa study the average price of aluminum is estimated to be $\$ 4.50 / \mathrm{kg}$, compared to $\$ 1.50 / \mathrm{kg}$ for copper. The total material costs become $\$ 5.7$ million for aluminum and $\$ 1.6$ million for the copper jacket.

Two final points are made about protection. First, the temperature integral of the specific heat divided by the resistivity should be recalculated for the copper-jacketed aluminum conductor. This would probably result in different 1 imits on $J$ and would require a second iteration to calculate $\rho_{A}$. Second, $J_{A}$ could be reduced significantly below $15 \mathrm{kA} / \mathrm{cm}^{2}$ with only a modest increase in conductor cost. For instance, if $\mathrm{J}_{\mathrm{A} 1}=10 \mathrm{kA} / \mathrm{cm}^{2}$, then $\mathrm{J}=10.9 \mathrm{kA} / \mathrm{cm}^{2}, \quad \rho=3.92 \times 10^{-8} \Omega \mathrm{cm}$, and $\rho_{A 1}=4.26 \times 10^{-8} \Omega \mathrm{cm} \quad(R R R=197)$. The cost for aluminum is virtually unchanged, whereas that for the copper jacket is increased by $\$ 0.5$ million. The incremental cost for the finished, installed conductor could be so small 
as to be more than canceled by a savings in the costs associated with protection.

\section{E. Conductor Stability}

A11 the numbers needed to demonstrate stability are now available. From Eq. $(A-3)$ the Joule heating for each quadrant of the 50-kA conductor is given by

$$
q_{J}=\frac{I^{2} \rho}{A}=\left(1.25 \times 10^{4}\right)^{2} \times 2.56 \times 10^{-8} /(0.928)^{2}=4.64 \mathrm{~W} / \mathrm{cm},
$$

whereas, from Eq. $(A-5)$ the allowable heat flux per centimeter of conductor length is

$$
q_{V}+q_{H}=5 f(w+t)=5(0.5)(0.928+0.928)=4.64 \mathrm{~W} / \mathrm{cm} \quad .
$$

From Eq. A-4 the surface heat flux will be the limiting condition provided the channel widths are given by

$$
x^{\prime \prime}=x^{\prime} \geq(2 / 3) w^{4 / 3}=0.60 \mathrm{~cm}
$$

\section{CONDUCTOR LOSSES}

\section{A. Hysteretic Loss}

During a charging cycle, the field at position $r$ within the conductor changes from $B_{\min }(\bar{r})$ to $B_{\operatorname{mix}_{i x}}(\bar{r})$. This results in an energy loss from hysteresis within the supercondacting filaments of diameter $d$ given by

$$
Q_{h}=\frac{2}{3 \pi} d \int \lambda(r) d V \int_{B_{\min }(\bar{r})}^{J_{c}(B) d B}\left[1+(I / I)^{2}\right],
$$

and a corresponding power loss, $P_{h}=Q_{h} / T$. The space factor, $\lambda$, is defined by

$$
\lambda=\frac{A_{\text {sc }}}{A_{\text {cond }}},
$$


and the volume integral is taken over the conductor volume rather than over only the superconductor volume. In most coils $\lambda=$ constant, and the volume integrals must be evaluated numerically. Here, however, the amount of superconductor has been arranged at every position so that $\lambda(\bar{r}) J_{c}(B)$ and, thus, $\left(I / I_{C}\right)$ are independent of position and equal to their values at the maximum field position. If these quantities are written as $\lambda_{M^{J} c}(B)$ and $\left(I / I_{C}\right)_{M}$ respectively, then Eq. $(A-10)$ becomes

$$
Q_{h}=\frac{2}{377} d \lambda_{M} V_{c} \int_{B_{\min }}^{B_{\max }} J^{J}\left[1+\left(I / I_{C}\right)_{M}^{2}\right],
$$

where the field integral now refers to the maximum field point, and $V_{c}$ is tha conductor volume. Note that $\lambda_{M} V_{C}$ is the superconductor volume which would have been present if the superconductor area had not been graded. The $J_{C}$ and $I_{C M}$ at any field can be written in terms of their specified values at $5.2 \mathrm{~T}$ from Table A-II and by using the Kim-Anderson formula

$$
J_{C}(B)=3.3 \times 10^{9}\left(\frac{5.2+B o}{B+B 0}\right) A / m^{2}
$$

and

$$
I_{C M}(B)=5.56 \times 10^{4}\left(\frac{5.2+B o}{B+B O}\right) A
$$

Also, at the maximum field point, $B=9.4 \times 10^{-5} \mathrm{I}$.

The integral may be evaluated analytically. Numerical values for $Q_{h}$ and the corresponding power, $P_{h}$, are given in Table A-V. These values depend somewhat upon $B_{0}$, which in turn seems to depend upon the detailed heat treatment schedule during wire fabrication. A relatively low value of $B_{0}$ $=0.20$ has been used, which leads to a conservatively large value of $Q_{h}$. If $B_{0}=1.0$ had been assumed, then $Q_{h}$ would have been reduced by $9 \%$.

\section{B. Self-Field Loss}

The filaments within a cable strand are fully transposed with respect to the total field generated by the magnet but are not transposed with respect to the self-field generated by the strand itself. This produces a nonuniform 
current distribution among these filaments and an additional source of hysteretic loss which, following wilson, is

$$
Q_{s}=n \frac{\mu_{0} I^{2}}{2 \pi^{2} D^{2}} V_{s}
$$

where $I$ and $D$ are the current and diameter of one strand in the cable and $V_{S}$ is the volume of the strands. See Table A-II. The $\eta$ depends upon the magnetic history of the specimen and upon ( $I / I_{C}$ ); it equals unity for the initial coil charge but is reduced to 0.1 for the second and subsequent chargedischarge cycles.

c. Coupling Loss

Coupling currents are eddy currents which flow longitudinally in the superconductor and transversely through the copper matrix. Because the rms value of the radial component, $B_{r}$, of the magnetic field is roughly one-third the rms value of the axial component, $B_{z}$, the loss can be minimized by placing the composite cable with its wide face vertical, that is, normal to $B_{r}$.

\section{TABLE $A-V$}

CALCULATED AVERAGE ELECTRICAL LOSSES IN THE 1-GWh COIL FOR THREE CONDITIONS

During 4-h Charge or Discharge

Hysteresis, Ph

Self-field, $P_{S}$

Coupling, $P_{C}$

Eddy currents, $P_{E}$ (conductor)

Eddy currents, $P_{E}$ (dewar)

Joints, PJ

While Holding Full Charge

$$
\begin{aligned}
& P_{J} \\
& P_{h}=P_{S}=P_{C}=P_{E}=P_{E}^{\prime}
\end{aligned}
$$

While Holding Minimum Charge

$$
P_{h}=P_{S}=P_{C}=P_{E}=P_{E}^{\prime}
$$

Watts

$$
215
$$

61 
A formula for this energy loss per unit volume of cable, $\Delta Q_{c 1} / \Delta V$, has been given by Wilson as

$$
\frac{\Delta Q_{c 1}}{\Delta V}=\frac{\Delta B_{r} \Delta \dot{B}_{r} p^{2}}{120 p_{c}}\left(\frac{W^{\prime}}{2}\right)^{2} \quad \frac{W^{\prime}}{t^{\prime}} .
$$

The cable has dimensions $w^{\prime}$ and $t^{\prime}\left(w^{\prime}>t^{\prime}\right)$, twist pitch $p$, and transverse resistivity $\rho_{C}$, whereas the coriductor is a square, $2 t$ on a side. The appropriate dimensions are given in Tables A-II and A-IV, whereas the value of $\rho_{c}$ appropriate to $25 \mu \mathrm{m}$ filaments has been measured as $\rho_{C}$ $=5 \times 10^{-10} \Omega \mathrm{m}$, almost independent of field. The axial fielo contribution similar to Eq.(A-13a) is negligible. There are, however, additional contributions from shunting currents which flow through the stabilizer. Formulae, derived by Turck $^{8}$ for these losses, are

$$
\frac{\Delta Q_{c 2}}{\Delta V}=\frac{\Delta B_{r} \Delta \dot{B}_{r} p^{2}}{24 \rho_{1}}
$$

and

$$
\frac{\Delta Q_{c 3}}{\Delta V}=\frac{\Delta B_{z} \Delta \dot{B}_{z} p^{2}}{128 \rho_{2}}\left(\frac{t^{\prime}}{w^{\prime}}\right)^{2},
$$

where $\Delta Q_{c 2} / \Delta V$ and $\Delta Q_{3} / \Delta V$ are the energy losses per unit volume caused by radial and axial fields, respectively, and $\rho_{1}$ and $\rho_{2}$ are effective resistivities of the current paths. Both $\rho_{1}$ and $\rho_{2}$ must be of the order of the stabilizer resistivity, $\rho$. The three components must be averaged over the coil volume and must include the magnetic field variation of the various resistivities. The three components make fractional contributions to the $f i-$ nal total of $0.58,0.41$, and 0.01 for Eqs. $(A-13 \mathrm{a}),(A-13 \mathrm{~b})$, and $(A-13 \mathrm{C})$, respectively.

D. Eddy Current Loss

Eddy current loss in the stabilizer is given approximately by

$$
\frac{\Delta Q_{E}}{\Delta V}=\frac{\Delta B \Delta \dot{B R}_{e}}{4 \rho},
$$


where $R_{e}$ is an effective radius of the stabilizer defined so that $\pi R_{e}^{2}$ $=A$ where $A$ is the cross-sectional area of the stabilizer. Again, Eq. $(A-14)$ must be averaged over the coil volume.

Eddy current losses in the coil structure represent, potentially, a far more serious problem. The eddy current loss in a ring of material of radius a and volume $V$ is given by

$$
P_{E}=\frac{\dot{\phi}^{2} v}{4 \pi^{2} a^{2} \rho}
$$

The $\phi$ is the total flux threading the plane of the ring, which may be calculated from the coil inductance by the reiation

$$
\phi=\frac{L I}{N}
$$

The resistivity of stainless steel at 1 to $4 \mathrm{~K}$ is $67 \times 10^{-8} \Omega \mathrm{m}$, and the volume of steel in the dewar is $650 \mathrm{~m}^{3}$, so that $P_{E}=1.8 \times 10^{4} \mathrm{~W}$. Thus each dewar must contain an insulating section so that it does not act like a 1 arge, conducting ring. In that case, the flux in Eq. $(A-15)$ becomes that which actually passes through the steel, and the formula reverts to that shown in Eq. (A-14) for each element of the dewar, with $R_{e}$ a characteristic dimension of that element. This may be the height, width, or thickness depending upon whether the dewar sides or top are considered and whether the calculation involves $B_{r}$ or $B_{z}$. The value given in Table $A-V$ is a very rough estimate. E. Joint Losses

Electrical joints will be necessary at both the inside and outside turns of the pancake coils, because a true double pancake would be very unwieldy to wind. There will be 857 joints including the end connections. At each joint half the stabilizer would be removed from each of the two conductors, the exposed composite cable would be soldered together with a 1-m overlap, and the conductor then would be mechanically clamped and braced. The current must transfer through two resistances in series.

1. The copper stabilizer in the composite strands, which is taken to have an RRR of 50 and an average path length equal to a strand diameter. 
2. The solder layer, which is assumed to have a thickness of $0.1 \mathrm{~mm}$ and a resistivity of $5 \times 10^{-9} \Omega \mathrm{m}$, corresponding to $60 \mathrm{~Pb}-40 \mathrm{Sn}$ solder.

With these assumptions the two contributions to the resistivity are equal. It is further assumed that each strand in the cable can transfer current over the entire length of the joint because of the shunting effect of the copperaluminum stabilizer. Each joint is then found to have a resistance of $6 \times 10^{-11} \Omega$. The resultant Joule heating must be averaged over the daily cycle of the coil.

F. Mechanical Losses

There are two possible, distinct sources of mechanical irreversibility in the proposed design. First, many elements are mechanically inhomogeneous, anisotropic systems with internal strains arising from fabrication and differential thermal contraction. They may show nonlinear stress-strain relations and hysteresis. Experiments show that 316 stainless steel exhibits no mechanical hysteresis provided it is cycled in the elastic range and that this is true for both annealed material and material containing $0.7 \%$ plastic strain. The proposed design keeps all the components well within their elastic limit, but it remains to be shown experimentally if the losses would in fact be zero in that case for all the materials. Second, mechanical losses may arise from the relative motion of various coil components with frictional effects, and the design should minimize such motion.

\section{COIL WINDING}

As presently conceived, the coil will be formed of 856 pancakes, each with five radial turns in thickness, with electrical joints at both the inner and outer radii. All pancakes will be wound from the outer radius to the inner radius, and successive pancakes must be alternately wound clockwise and counterclockwise. Radial spacers will be placed as each pancake is wound; axial spacers will be laid down before winding a subsequent pancake. An advantage of the proposed conductor is that it may be completely assembled in a factory and shipped to the winding site on a spool. This simplifies the necessary underground winding machinery and facilitates the task of grading the superconductor. Five turns of assembled conductor will have a mass of 3.32 metric tons and a length of $2.1 \times 10^{3} \mathrm{~m}$. The material should fit comfortably on a 4-m-diameter, 1-m-high winding spool as four layers with a totai radial build of $7.5 \mathrm{~cm}$. The spools themselves must be carefully wound with attention paid 
to the layer-to-1ayer transitions at the ends. The winding machinery must be capable of supporting the spool, providing appropriate tension, restraightening the conductor, forming the conductor to the proper ripple radius, and accurately guiding the conductor to the desired location. The winding machine can run on a track which later becomes part of the dewar structure.

If the conductor can be laid at $1 \mathrm{~m} / \mathrm{min}$, the winding task takes $3.0 \times 10^{4} \mathrm{~h}$, or $3.4 \mathrm{yr}$ of around-the-clock operation. This is almost certainly too long, because winding should proceed rapidly enough to stay ahead of the crews welding the dewars and installing plumbing, struts, and thermal insulation. Therefore, either the winding speed will have to be three to five times faster or several coil segments will have to be wound simultaneously.

If there is a crew of three per winding machine plus $50 \%$ for supervision, joint fabrication, and miscellaneous tasks, the job is found to take $1.35 \times 10^{5}$ man-hours at $1 \mathrm{~m} / \mathrm{min}$ per crew. This effort costs $\$ 2.7$ million at $\$ 20$ per man-hour.

\section{CONDUCTOR SUPPORT}

If a single conductor is loaded axially, the vertical copper sidewalls bow outward; and the resultant bending stress limits the load-bearing capability of the conductor. In a coil, five conductor turns are laid side by side with intermittent radial spacers, so that adjacent turns provide support against bowing for all except the innermost and outermost walls of the entire pancake. In support Option I, Fig. A-4, a thick band of support material is to be wound along these two surfaces to prevent bowing and also to help support the axial load. Once bowing is el iminated, the conductor can be designed for either maximum compression in the copper or maximum strain in the aluminum. This design is for a strain of $10^{-3}$ in the aluminum stabilizer, in which case the conductor will support $84 \mathrm{MPa}(12.2 \mathrm{ksi})$ with a stress in the copper of $134 \mathrm{MPa}(19.4 \mathrm{ksi})$.

Axial load is transmitted from pancake to pancake by spacer bars placed at the same angular increments as the radial spacer blocks and spanning the entire radial thickness of the pancake including the inner and outer support bands. Because the axial load accumulates within a dewar section, the amount of support material can also vary with position. Dimensions and stresses given immediately below are for the most heavily loaded pancakes. The axial 
load on a pancake is shared by the conductor, radial spacer blocks, and support bands acting in parallel. The support elements must be high-modulus material to carry a significant share of the load; thus $G-10 C R$ is not a suitable material. Stainless steel bands and blocks suitably covered with electrically insulating material are proposed. If the spacers are $0.6 \mathrm{~cm}$ thick radially and cover $50 \%$ of the conductor surface, then the support bands must total $5.0 \mathrm{~cm}$ in thickness. The outer band should be made much thicker than the inner one, so that it can share the tensile load. The compressive stress in the band will be $200 \mathrm{MPa}(29.0 \mathrm{ksi})$.

The axial spacer bars are really just space fillers. Aluminum alloy, again covered with an electrical insulator, would seem to be the least costly material available for this service. For bars covering $50 \%$ of the pancake area, the compressize stress in the aluminum will be $240 \mathrm{MPa}$ ( $35 \mathrm{ksi}$ ).

Table A-VI gives the volumes and fabricated prices for the various support elements. The cost of electrical insulation is not included. The rightmost column gives the cost for a graded structure, which is estimated to be $70 \%$ of an ungraded one.

TABLE A-VI

VOLUME AND COST OF CONDUCTOR SUPPORT ELEMENTS

OPTION I

\begin{tabular}{|c|c|c|c|c|c|}
\hline El ement & Material & $\begin{array}{c}\text { Unit Cost } \\
\$ / \mathrm{kg} \\
\end{array}$ & Volume, $\mathrm{m}^{3}$ & $\begin{array}{l}\text { Costa } \\
\$ 10^{6}\end{array}$ & $\begin{array}{l}\text { Costb } \\
\$ 10^{6} \\
\end{array}$ \\
\hline $\begin{array}{l}\text { Radial spacer } \\
\text { Support band } \\
\text { Axial spacer }\end{array}$ & $\begin{array}{l}\text { S.S. } \\
\text { S.S. } \\
\text { Ai alloy }\end{array}$ & $\begin{array}{l}2.50 \\
2.50 \\
2.50\end{array}$ & $\begin{array}{r}118 \\
331 \\
1040 \\
\text { TOTAL }\end{array}$ & $\begin{array}{r}2.3 \\
6.5 \\
7.1 \\
15.9\end{array}$ & $\begin{array}{r}2.3 \\
4.5 \\
5.0 \\
11.8\end{array}$ \\
\hline \multicolumn{6}{|l|}{ OPTION II } \\
\hline $\begin{array}{l}\text { Radial spacer } \\
\text { Support band } \\
\text { Axial spacer } \\
\text { Axial support }\end{array}$ & $\begin{array}{l}\text { A1 alloy } \\
\text { A1 alloy } \\
\text { A1 alloy } \\
\text { S.S. }\end{array}$ & $\begin{array}{l}2.50 \\
2.50 \\
2.50 \\
2.50\end{array}$ & $\begin{array}{r}118 \\
146 \\
867 \\
14 \\
\text { TOTAL }\end{array}$ & $\begin{array}{l}0.8 \\
1.0 \\
5.9 \\
0.3 \\
8.0\end{array}$ & $\begin{array}{l}0.8 \\
1.0 \\
4.1 \\
0.3 \\
6.2\end{array}$ \\
\hline
\end{tabular}

assumes equal structure at all positions. bstructure varies with position. 
Although support Option I is straightforward to construct, it is rather expensive. Support Option II (Fig. A-4) can reduce the cost of internal structure by a large amount, although it adds complexity and an unknown cost to the dewar. The conductor stack is divided in half vertically, with the lower half resting on the dewar end and the upper half resting on a ledge attached to both the inner and outer helium vessel walls. The total force or. each half-stack is now only slightly larger than the conductor itself can support. The bands and radial spacers $c$ an be made of aluminum alloy, coated with insulation, at a stress level of $73 \mathrm{MPa}(11 \mathrm{ksi})$. The 14 layers of axiai supports which rest directly upon the ledges must support a shear stress of $400 \mathrm{MPa}$ (5ó ksi), so they are made of steel rather than aluminum. Only an inner band is needed, since the outer helium vessel wall also acts as a support.

\section{REFERENCES}

1. R. H. Boom, Ed., "Wisconsin Superconductive Energy Storage Project," Vol. I and II, Engineering Experiment Station, College of Engineering, University of Wisconsin reports (1974 and 1976).

2. H. V. Hassenzahl, Ed., "Progress Report Superconducting Magnetic Energy Storage Project," Los Alamos Scientific Laboratory report LA-5472-PR (1973).

3. J. D. Rogers, W. V. Hassenzahl, and R. I. Schermer "1-GWh Diurna? Load-Leveling Superconducting Magnetic Energy Storage System Reference Design," Los Alamos Scientific Laboratory report LA-7885-MS Vol. I (1979).

4. C. N. Cochran, R. K. Dawless, and J. B. Whitchurch, "1-Gwh Diurnal Load-Leveling Superconducting Magnetic Energy Storage System Reference Design; Appendix B: Cost Study, High-Purity Aluminum Production," Los Al amos Scient if ic Laboratory report LA-7885-MS Vol. III (1979).

5. A. Petrovitch, M. S. Walker, B. A. Zeitlin, and J. D. Scudiere, "Final Report, Conductor for a 10-MWh Superconducting Magnetic Energy Storage Coil," IGC report 577-1, Intermagnetics General Corporation, Guilder land, NY, 1977 .

6. H. R. Segal, "Reinforced Aluminum as a Superconducting Magnet Stabilizer," IEEE Trans. Magn. Mag-13, 109-11 (1977).

7. F. R. Fickett, "Magnetoresistance of Very Pure Polycrystalline Aluminum," Phys. Rev. B3, 1941 (1971).

8. B. Turck, "Losses in Superconducting Multifilament Composites under Alternating Changing Fields," Los Alamos Scientific Laboratory report LA-7639-MS (1979). 


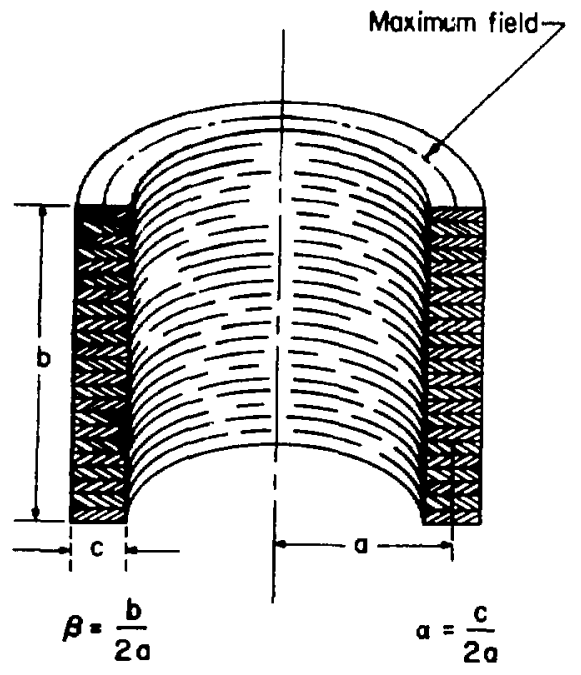

Fig. A-1.

Geometric variables of a simple solenuid.

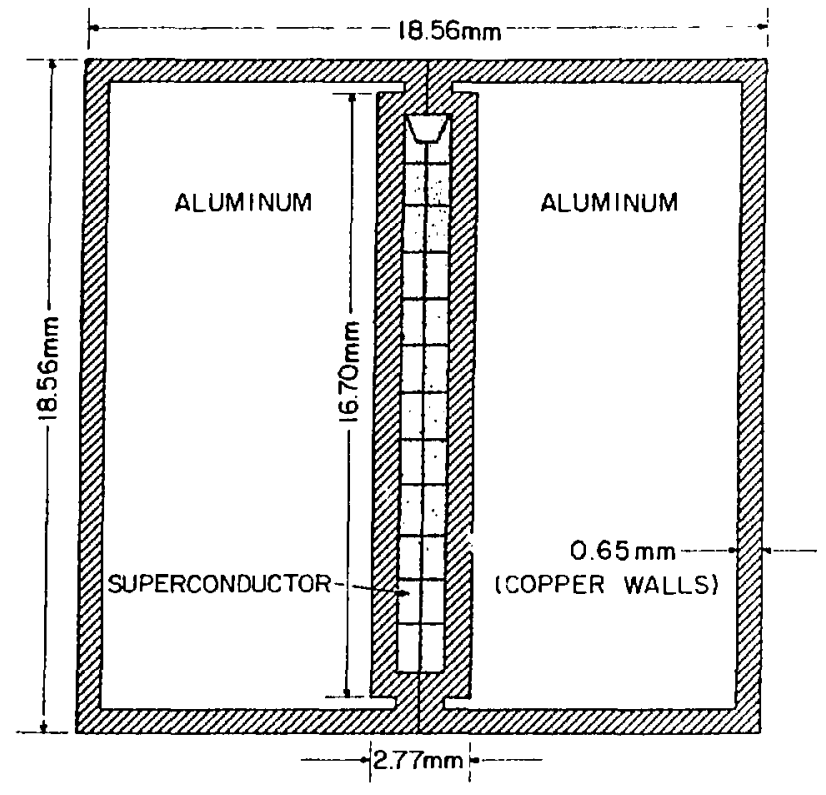

Fig. $A-3$.

Proposed 50-kA al umi num-s tabilized copper-jacketed conductor.
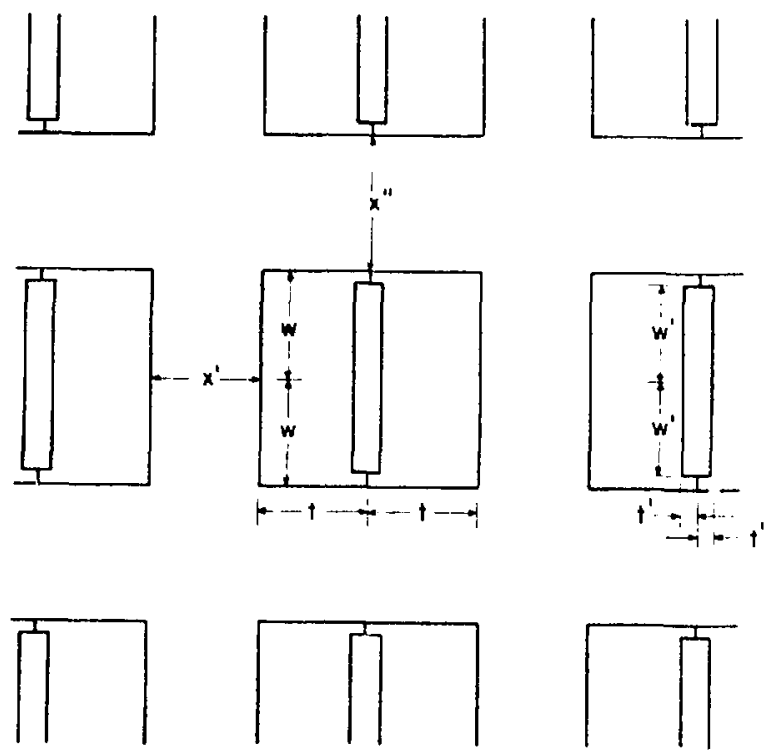

Fig. A-2.

Cross section of a conductor array, showing geometric variables.

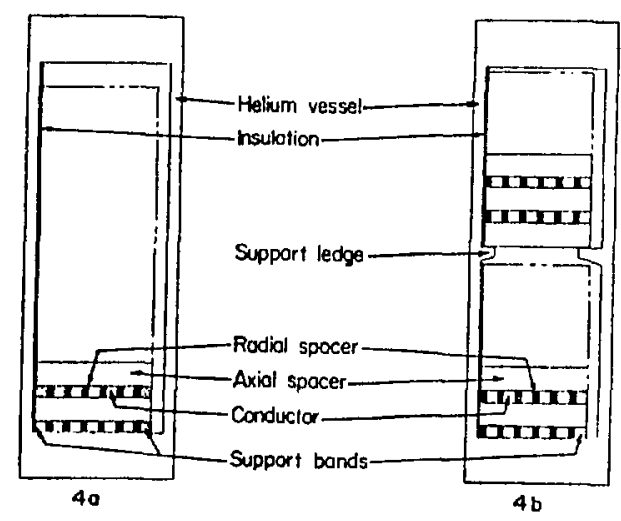

Fig. A-4.

Conductor support options. In Option I (left), the axial load accumulates to the bottom of the helium vessel. In Option II (right), half of the axial load is taken by the ledges. 